\title{
Venous thromboembolism during pregnancy and postpartum: study in a tertiary hospital
}

\begin{abstract}
Objective: To examine whether implementing an evidence-based guideline reduces the risk of symptomatic Venous Thrombo Embolism (VTE) during pregnancy and up to 3 months postpartum.
\end{abstract}

Methods: This was a hospital-based retrospective cohort study of 9,041 deliveries over a 16-year period at Nara Medical University Hospital, Japan. We compared the incidence of symptomatic VTE before (1998-2007) and after (2008-2013) the introduction of the Japanese thrombo prophylaxis guideline and analyzed the medical records of pregnant women admitted to this hospital.

Result: The overall incidence of symptomatic VTE among the study subjects was $0.28 \%$ (25/9,041 deliveries), representing an incidence of Deep Venous Thrombosis (DVT) and Pulmonary Thrombo Embolism (PTE) of $0.22 \%$ and $0.05 \%$, respectively. During the pregnancy and postpartum periods, 19 and 6 VTE cases, respectively, were observed. The risk of DVT was possibly the highest in the first trimester. All the objectively confirmed PTEs typically presented postpartum or within 26 days of delivery. Further, $96 \%$ of VTE cases had one or more large anamnestic risk factor(s) The effect was small, but the incidence of VTE $(0.40 \%$ vs $0.14 \%)$ decreased by $65 \%$ during the post-implementation period

Conclusion: VTE cases may at least be partially prevented using the clinical practice guideline.

Keywords: deep vein thrombosis, pulmonary embolism, pregnancy, postpartum thrombosis, thrombo prophylaxis
Volume I Issue I - 2016

\author{
Ryuji Kawaguchi, Shoji Haruta, Hiroshi \\ Kobayashi \\ Department of Obstetrics and Gynecology, Nara Medical
} University, Japan

Correspondence: Ryuji Kawaguchi, Department of Obstetrics and Gynecology, Nara Medical University, Japan, Tel +8I-744-298877, Fax+8I-744-23-6557, E-mail kawaryu@naramed-u.ac.jp

Received: August 12, 2016 | Published: August 30, 2016
Abbreviations: VTE, venous thrombo embolism; DVT, deep vein thrombosis; PTE, pulmonary thrombo embolism

\section{Introduction}

Venous thrombo embolism (VTE), including deep venous thrombosis (DVT) and pulmonary thrombo embolism (PTE), remains a major public health problem with a high mortality worldwide. The hyper coagulable state of pregnancy is possibly associated with an increasing risk of VTE. ${ }^{1}$ Major predisposing factors are increased maternal age, obesity, cesarean delivery, immobilization because of threatened abortion and threatened premature delivery, dehydration because of hyper emesis, heart disease, and a history of thrombosis and thrombophilia. ${ }^{2,3}$ Based on this knowledge, the Japanese medical establishment implemented a risk score model (the Japanese VTE thrombo prophylaxis guideline) for obstetricians. ${ }^{4}$ However, physicians still fail to prescribe prophylaxis for patients at risk of VTE, possibly because of the uncertainty regarding risk factors and difficulty in recognizing individuals at risk. ${ }^{5}$

In our hospital, evidence-based Japanese prophylaxis guideline recommendations for the prevention of VTE during pregnancy and postpartum were implemented in 2008. Clinical data, including demographics, co morbidities, adverse events, the time of VTE diagnosis, incidence, and identification of the risk factors for VTE, were prospectively collected and retrospectively pooled for VTE analysis. A cohort study was conducted to investigate the incidence of VTE during pregnancy and postpartum before and after the introduction of the prophylaxis guideline in a single tertiary hospital. We revealed a marked reduction in the incidence of VTE before and after the implementation of the guideline.

\section{Materials and methods}

We searched the number of thrombo embolic events during pregnancy and up to 3 months postpartum at a tertiary academic medical center, Department of Obstetrics and Gynecology, Nara Medical University Hospital, Nara, Japan. VTE was defined as a symptomatic DVT or PTE. Patients were consecutively approached for participation in the study between January 1998 and December 2013. Follow-up lasted until 3months after delivery. During the study period, 9,041 deliveries were performed. Patients with DVT were largely confirmed or objectively excluded by complete compression ultrasound. Moreover, PTE was diagnosed by a computerized tomography (CT) angiography. Contrast-enhanced chest CT revealed filling defects of the pulmonary artery as a direct sign of PTE. The ultrasonography and CT results were interpreted by a minimum of two expert radiologists. Their data were extracted from the medical charts and recorded on case report forms; additional data were collected by physical examination, blood samples, and questionnaires. Questions regarding the known risk factors for VTE, such as age, parity, co morbidity, obstetric complications, mode of delivery, history of using oral contraceptive pills, thrombophilia, past history, family history of VTE, objectively documented thrombotic and major bleeding events, and type of prophylaxis, were included. Thrombophilia testing, including clotting profile and natural anticoagulants (anti thrombin, protein $\mathrm{C}$, 
and protein S), was performed as a part of the study. This protocol was approved by the Ethics Committee of the Faculty of Medicine, Nara Medical University.

The overall study period was divided into two time intervals: before the introduction of the guideline (1998-2007) and after the introduction, representing the adoption phase (2008-2013). Before the implementation of the guideline-based VTE risk assessment, patients basically received mechanical prophylaxis, including the use of elastic stockings (ES) and intermittent pneumatic compression (IPC). Early ambulation was also recommended. In 2007, the multidisciplinary working group in this hospital adopted the Japanese VTE prophylaxis guideline published in 2004; this guideline is available at http://www. jsth.org/publications/pdf/tokusyu/19_1.012.2008.pdf. Thrombo prophylaxis included both mechanical and pharmacological mechanisms. Candidates for either prophylactic or therapeutic anticoagulation during pregnancy include women with a history of thrombosis or those who are at significant risk of venous thrombo embolism during pregnancy or the postpartum period, such as those with high-risk acquired or inherited thrombophilias. A nearly mobilization and the use of ES and IPC were the most common mechanical prophylaxes. Pharmacological anticoagulants included unfractionated heparin (UFH) and low molecular weight heparin (LMWH). The mean duration of prophylaxis was 5days, and the prophylactic heparin dose was standardized. From 2008 to 2009, the standard treatment included activated partial thromboplastin time-adjusted UFH. Most patients in our hospital were initially treated with LMWH, predominately with two 2,000-U injections daily as it was the drug of choice in the most recent period (2010-2013). The main outcome measure was the trend of incidence of symptomatic VTE.

Participants in the screening were registered in a computer system and various data were collected. The data were analyzed using the SAS program for microcomputers, and statistical significance was set at $\mathrm{P}<0.05$. Differences between observed results were checked by the Chi 2 test; differences between means were checked by the t-test and analysis of variance.

\section{Results and discussion}

\section{Results}

In this study, 25women had symptomatic VTE during the 16-year study period (Table 1). Among the 25patients, 11 with VTE were initially observed at Nara Medical University Hospital (in-hospital group).After being diagnosed with VTE in other hospitals, 14 patients were transferred to our hospital (transfer group). The data were analyzed for all patients, including the in-hospital and transfer groups, and also for those who excludes transfer group. A total of 9,041 deliveries occurred during the study period with 4549 during the first and 4942 during the second study period. All VTEs were verified by objective methods: ultrasound $(\mathrm{n}=20), \mathrm{CT}(\mathrm{n}=7)$, and ventilation/perfusion scintigraphy $(n=2)$. Many patients were examined by more than one method; all patients were then admitted to the hospital for initial therapy.

Table I Comparison of the Clinical and Laboratory Characteristics of Japanese Patients with Symptomatic VTE

\begin{tabular}{|c|c|c|c|c|}
\hline \multirow[b]{2}{*}{ Time intervals } & \multicolumn{2}{|l|}{ DVT } & \multicolumn{2}{|l|}{ PE } \\
\hline & From I 998 to 2007 & From 2008 to 2013 & From I 998 to 2007 & From 2008 to 2013 \\
\hline No. of Cases & 13 & 7 & 5 & 0 \\
\hline In- Hospital & 5 & I & 5 & 0 \\
\hline Transfer & 8 & 6 & 0 & 0 \\
\hline Age (Mean+/- SD) & $31.8+/-2.9$ & $31.5+/-6.3$ & $37.8+/-3.6$ & - \\
\hline \multicolumn{5}{|l|}{ Parity } \\
\hline Nulliparous & 3 & 2 & 2 & - \\
\hline Parous & 10 & 5 & 3 & - \\
\hline \multicolumn{5}{|l|}{ BMI } \\
\hline$<25 \mathrm{~kg} / \mathrm{m}^{2}$ & 13 & 5 & 3 & - \\
\hline $25-30$ & 0 & 2 & I & - \\
\hline$>30$ & 0 & 0 & I & - \\
\hline \multicolumn{5}{|l|}{ Pregnancy-related condition } \\
\hline Threatened Abortion & 3 & I & 0 & - \\
\hline Hyper Emesis & 5 & 4 & 0 & - \\
\hline $\begin{array}{l}\text { Threatened Premature } \\
\text { Delivery }\end{array}$ & 2 & I & 2 & - \\
\hline Diabetes & 0 & 0 & 0 & - \\
\hline Preeclampsia & 0 & 0 & 3 & - \\
\hline \multicolumn{5}{|l|}{ Delivery } \\
\hline Abortion & 2 & 0 & 0 & - \\
\hline Delivered By Cesarean & 7 & 0 & 4 & - \\
\hline Delivered Vaginally & 4 & 7 & l & - \\
\hline
\end{tabular}


Table Continued...

\begin{tabular}{|c|c|c|c|c|}
\hline & DVT & & PE & \\
\hline Time intervals & From I 998 to 2007 & From 2008 to 2013 & From I998 to 2007 & From 2008 to 2013 \\
\hline \multicolumn{5}{|l|}{ During pPregnancy } \\
\hline The First Trimester & 9 & 4 & 0 & - \\
\hline The Second Trimester & 0 & 3 & 0 & - \\
\hline The Third Trimester & 3 & 0 & 0 & - \\
\hline In the postpartum & I & 0 & 5 & - \\
\hline \multicolumn{5}{|l|}{ Clinical signs and symptoms } \\
\hline Swelling & 4 & 4 & 0 & - \\
\hline $\begin{array}{l}\text { Difficulty Walking or } \\
\text { Extremity Discomfort }\end{array}$ & 7 & 7 & 0 & - \\
\hline Erythematic & I & 0 & 0 & - \\
\hline Shock & 0 & 0 & 3 & - \\
\hline Sudden Dyspnea & 0 & 0 & 1 & - \\
\hline Syncope & 0 & 0 & 2 & - \\
\hline \multicolumn{5}{|l|}{ The sites of DVT } \\
\hline $\begin{array}{l}\text { The Left Lower } \\
\text { Extremity }\end{array}$ & 9 & 5 & 1 & - \\
\hline $\begin{array}{l}\text { The Right Lower } \\
\text { Extremity }\end{array}$ & 3 & 1 & 0 & - \\
\hline Bilateral & 1 & 1 & 0 & - \\
\hline Ileac Veins & 7 & 3 & 0 & - \\
\hline Femoral Veins & 7 & 4 & I & - \\
\hline Cural Veins & 5 & 4 & 0 & - \\
\hline \multicolumn{5}{|l|}{ Thrombophilia } \\
\hline Protein S Deficiency & 2 & 0 & 0 & - \\
\hline Protein C Deficiency & 2 & 0 & 0 & - \\
\hline Protein in Deficiency & 3 & 2 & 0 & - \\
\hline
\end{tabular}

VTE, Venous Thromboembolism; BMI, Body Mass Index; PE, Pulmonary Embolism; DVT, Deep Vein Thrombosis

We compared the clinical characteristics between patients with DVT and those with PTE. Characteristics of the study patients are summarized in Table 1. Of the 25 VTE cases, 20(80\%) were DVT and $5(20 \%)$ were PTE, representing an incidence of $0.22 \%$ and $0.05 \%$, respectively. One PTE case was fatal. The incidence of PTE was lower than that of DVT $(0.05 \%$ vs. $0.22 \%)$. For VTE diagnosis, DVT events occurred at an early stage in pregnancy, while the period of the highest PTE risk was in the weeks after delivery. Of the 20 DVTs, $65 \%(n=13)$ of ante partum DVT occurred during the first trimester, $12 \%(n=3)$ in the second trimester, $12 \%(n=3)$ in the third, and $4 \%$ $(n=1)$ in the postpartum period. All PTEs occurred in the postpartum period and within 26days of delivery. The age at the time of diagnosis of VTE ranged from 21 to 43years (mean, 32.9 \pm 4.8 ). Furthermore, the mean age at the time of diagnosis of VTE was significantly different between the DVT and PTE groups: $31.8 \pm 4.2$ years in the DVT group and 37.8 \pm 3.6 years in the PTE group $(\mathrm{P}=0.008)$. Eighteen patients $(72 \%)$ were porous $(15 / 20 ; 75 \%$ for DVT and $3 / 5 ; 60 \%$ for PTE) and $8(28 \%)$ were nulliparous $(5 / 20 ; 25 \%$ for DVT and $2 / 5 ; 40 \%$ for PTE). Three $(12 \%)$ were overweight (BMI of $>25 \mathrm{~kg} / \mathrm{m}^{2}$ and $<30 \mathrm{~kg} / \mathrm{m}^{2}$ ) and $1(4 \%)$ was obese $\left(\mathrm{BMI}\right.$ of $\left.>30 \mathrm{~kg} / \mathrm{m}^{2}\right)$. Nineteen patients $(76 \%)$ had been immobilized for more than 1 month, presumably because of pregnancy-related complications, including threatened abortion $(n=4)$, hyper emesis $(n=9)$, and threatened premature delivery $(n=6)$. Among the 5 patients with PTE, the incidence of pre-eclampsia was $60 \%(3 / 5)$. Pregnancy-induced hypertension and pre-eclampsia were more frequently observed in patients with PTE. Of the PTE patients, $4(80 \%)$ were delivered by cesarean and $1(20 \%)$ was delivered vaginally.

The mean duration of time from symptom onset to diagnosis was $6.9 \pm 8.4$ days (range, 0-29) during pregnancy and $0.7 \pm 1.6$ days postpartum. The mean duration from onset to therapy in the 5 patients with PTE was 0 days, which was much shorter than that in the 20 patients with DVT (mean duration, $6.8 \pm 8.2$ days; $\mathrm{P}=0.002$ ). Although clinical signs and symptoms of DVT are unreliable, the most common symptoms were difficulty in walking or extremity discomfort, which occurred in $16(84.2 \%)$ of the 19 pregnant women. Additional symptoms included swelling in 7(36.8\%) of the 19pregnant women and in $1(100 \%)$ postpartum woman. The most useful features for PTE diagnosis were shock $(60 \% ; 3 / 5)$, syncope $(40 \% ; 2 / 5)$, and sudden dyspnea $(20 \% ; 1 / 5)$. The mean hospital stay was longer in the PTE group 
(147.6 \pm 252.1 days) than the DVT group (55.2 \pm 40.5 days); however, this difference was not significant $(\mathrm{P}=0.131)$.

The sites of DVT are shown in Table 1. In all, DVT in 14/20 patients $(70 \%)$ involved the left lower extremity. DVT also occurred in the right leg in 4 patients $(20 \%$ and bilaterally in $2(10 \%)$. Overall, DVT in 16patients $(80 \%)$ involved the left lower extremity, whereas DVT in 6 patients $(30 \%)$ involved the right. Furthermore, DVT involved the iliac $(n=10)$, femoral $(n=11)$, or crural veins $(n=9)$. Ten of the pregnant patients $(50 \%)$ experienced pelvic vein DVT. No patient experienced concomitant PTE.PTE was mostly observed in the bilateral lobe pulmonary artery $(80 \%, 4 / 5)$; however, in 1 patient, it was observed in the left lobe pulmonary artery. Coagulation tests for thrombophilia were conducted in 20 of the 25 patients with VTE $(80 \%)$. Seven patients (35\%were diagnosed with thrombophilia; of which, 2 had protein S deficiency and 5 had antithrombin deficiency. The most common DVT risk factors were prolonged immobility $(85 \% ; 17 / 20)$, dehydration $(45 \% ; 9 / 20)$, and thrombophilia $(35 \% ; 7 / 20)$. A past VTE history was present only in the DVT group $(5 \% ; 1 / 20)$ and not the

\section{PTE group.}

The secondary objective was to compare the rate of symptomatic VTE before and after the implementation of the VTE prophylaxis guideline. Data from the pre-intervention (1998-2007) and post-intervention periods (2005-2013) were compared to evaluate the impact of the recommendations on the appropriateness of prophylaxis (Table 2). The post-intervention period revealed an incidence of VTE (DVT, $0.14 \% ; 7 / 4,942$ and PTE $0 \% ; 0 / 4,942)$ lower than the pre-intervention period (DVT, 0.28\%; 13/4,549 and PTE 0.11\%; 5/4,549).

The incidence of VTE decreased in the post-implementation period. Symptomatic VTE was significantly reduced by $65 \%$ using thrombo prophylaxis $(0.40 \%$ vs. $0.14 \%$; $\mathrm{P}=0.0254)$. Our data also showed that in hospitalized patients, the incidence of VTE decreased in the post-implementation period $(0.22 \% ; 10 / 4,549$ vs. $0.02 \%$; $1 / 4,942 ; \mathrm{P}=0.0047)$. The use of prophylaxis reduced the number of symptomatic VTE events by $65 \%$ [95\% confidence incidence (CI), 0.126-0.897] compared with no prophylaxis. This trend remained lower in the in-hospital group versus the transfer group.

Table 2 The impact of incidence of VTE before and after the implementation of the Japanese prophylaxis guidelines

\begin{tabular}{lll} 
& $\begin{array}{l}\text { Before the implementation of the VTE } \\
\text { prophylaxis guideline (1998-2007) }\end{array}$ & $\begin{array}{l}\text { After the implementation of the VTE prophylaxis } \\
\text { guideline (2008-20 I3) }\end{array}$ \\
\hline No. of Deliveries & 4,549 & 4,92 \\
No. of VTE & 18 & 7 \\
Incidence of VTE & $0.40 \%$ & $0.14 \%$
\end{tabular}

VTE,Venous Thromboembolism

\section{Discussion}

Although this study was a relatively small-sized hospital-based screening, we consecutively collected data for 16 years on symptomatic VTE, including lethal PTE. During 1998-2007, the patients received suboptimal prophylaxis. Some of the high-risk to very high-risk patients did not receive pharmacological VTE prophylaxis. We observed a $65 \%$ decrease in the incidence of symptomatic VTE episodes following the implementation of the risk-based clinical practice guideline for VTE prophylaxis (from $0.40 \%$ to $0.14 \% ; \mathrm{P}=0.0025$ ). Further, a significant improvement was observed in the incidence of VTE in hospitalized patients receiving an appropriate pharmacological VTE prophylaxis following the intervention (from $0.22 \%$ to $0.02 \% ; \mathrm{P}=0.0047$ ). Pharmacological VTE prophylaxis was associated with a significant reduction in the risk of symptomatic PTE by $100 \%$ ( $\mathrm{P}=0.0025 ; 95 \%$ CI, 0.000-1.004)

Our data revealed a high incidence of DVT in early pregnancy, an increased incidence of left-sided DVT, and the susceptibility of patients to PTE after cesarean delivery, which are consistent with those of previous reports. ${ }^{6}$ The predilection for the left leg has been previously reported, ${ }^{7,8}$ and anatomic reasons have been postulated. Altogether, $80 \%$ of pregnancy-associated VTE was DVT and $20 \%$ was PTE, consistent with the findings of a meta-analysis. ${ }^{7}$ Two-thirds of our DVT cases occurred in the first trimester. James et al. have reported that more patients suffer from DVT in early pregnancy than in the puerperium period. ${ }^{7}$ the frequency of DVT in the first trimester can be associated with early pregnancy problems, such as a longer immobilization because of hyper emesis or threatened abortion. In patients with an increased VTE risk, prophylaxis should be initiated early in pregnancy for reducing thrombotic events. In contrast, all PTE events occurred postpartum, and 4 of 5 cases occurred after the cesarean section. Our results were consistent with those of with a previous report, wherein the incidence was 5times higher among postpartum women than pregnant women. ${ }^{6}$

The present study identified risk factors that can be assessed by anamnesis and thrombophilia testing. We report that risk factors, such as hyper emesis, immobility, and inherited and acquired thrombophilia, appear to be very important for DVT. Elder women (>35years) and patients with a high body mass index, hypertension (possibly associated with pre-eclampsia), immobility, or who had undergone recent surgery (cesarean delivery) were at a higher risk of PTE. ${ }^{9,10}$ These are usually considered as established risk factors.

Advanced maternal age, particularly over 35years, may be an established risk factor for PTE, possibly due to an age-related increase in the frequency of cesarean section..$^{11,12}$ In our study, the risk of PTE, but not DVT, increased with age.

One of the 25 women (4\%) had a history of VTE. A past personal or family history of thrombosis appeared to be an important risk factor for recurrent events in pregnancy or postpartum. ${ }^{13}$ despite limited resources, thrombophilias were screened. Among the 20 patients with symptomatic DVT, 2 were protein S deficient and 5 were antithrombin deficient. Moreover, $35 \%$ of the pregnant women with DVT were later diagnosed with thrombophilia. Deficiencies of the natural anticoagulants (protein $\mathrm{S}$, protein $\mathrm{C}$, and antithrombin) are the main inherited risk factors and may be the predominant thrombophilias in Asia. ${ }^{14}$ As the protein $\mathrm{S}$ expression is down regulated by estrogen, an initial decrease in the protein $\mathrm{S}$ level was observed during pregnancy. 
The episodes of VTE in these patients occurred before middle age and the risk of VTE recurrence was high. There may be an indication for efficient thrombo prophylaxis among women with deficiencies of natural anticoagulants.

The finding of a lower VTE incidence during the guideline adoption period (2008-2013) may be attributed to the use of the algorithm in reducing the incidence of VTE. The decision on the indication and timing of prophylactic anticoagulation in pregnant women remains a major challenge, ${ }^{15-17}$ as there have been no large, randomized trials of thrombo prophylaxis in the pregnancy and postpartum periods, optimal protocols for thrombo prophylaxis have yet to be developed.

\section{Conclusion}

The implementation of the guideline may at least partially reduce the risk of the development of symptomatic VTE during pregnancy and postpartum. Considering the risk factors presented in this study, such as hyper emesis, immobility, and inherited thrombophilia, severe VTE cases may be preventable.

\section{Acknowledgements}

We thank all the study participants for their time and effort. We also thank Mikiko Kita for editorial assistance.

\section{Conflict of interest}

Author declares that there is no conflict of interest.

\section{References}

1. James AH. Venous thromboembolism in pregnancy. Arterioscler Thromb Vasc Biol. 2009;29(3):326-331.

2. Lindqvist PG, Torsson J, Almqvist A, et al. Postpartum thromboembolism severe events might be preventable using a new risk score model. Vasc Health Risk Manag. 2008;4(5):1081-1087.

3. James AH, Jamison MG, Brancazio LR, et al. Venous thromboembolism during pregnancy and the postpartum period: incidence, risk factors, and mortality. Am J Obstet Gynecol. 2006;194(5):1311-1315.

4. Sakon M. Postoperative pulmonary thromboembolism and its prevention from the perspective of the establishment of Japanese guidelines. Nihon Geka Gakkai Zasshi. 2005;106(3):237-240.
5. Gader AA, Haggaz AE, Adam I. Epidemiology of deep venous thrombosis during pregnancy and puerperium in Sudanese women. Vasc Health Risk Manag. 2009;5(1):85-87.

6. Heit JA, Kobbervig CE, James AH, et al. Trends in the incidence of venous thromboembolism during pregnancy or postpartum: a 30-year population-based study. Ann Intern Med. 2005;143(10):697-706.

7. James AH, Tapson VF, Goldhaber SZ. Thrombosis during pregnancy and the postpartum period. Am J Obstet Gynecol. 2005;93(1):216-219.

8. O'Connor DJ, Scher LA, Gargiulo NJ, et al. Incidence and characteristics of venous thromboembolism disease during pregnancy and the postnatal period: a contemporary series. Ann Vasc Surg. 2011;25(1):9-14.

9. Simpson EL, Lawrenson RA, Nightingale AL, et al. Venous thromboembolism in pregnancy and the puerperium: incidence and additional risk factors from a London perinatal database. BJOG. 2001;108(1):56-60.

10. James AH. Pregnancy-associated thrombosis. Hematology Am Soc Hematol Educ Program. 2009;277-285.

11. Lindqvist P, Dahlbäck B, Marŝál K. Thrombotic risk during pregnancy: a population study. Obstet Gynaecol. 1999;94(4):595-599.

12. Martineau M, Nelson-Piercy C. Venous thrombo embolic disease and pregnancy. Postgrad Med J. 2009;85(1007):489-494.

13. Benson MD. Pulmonary embolism in pregnancy. Consensus and controversies. Minerva Ginecol. 2012;64(5):387-398.

14. Angchaisuksiri P. Venous thromboembolism in Asia-an unrecognised and under-treated problem? Thromb Haemost. 2011;106(4):585-590.

15. Tufano A, Di Capua M, Coppola A, et al. The challenge of diagnosing pulmonary embolism in children, pregnant women, and elderly patients: a descriptive review of the literature. Semin Thromb Hemost. 2011;37(8):908-917.

16. Bagaria SJ, Bagaria VB. Strategies for Diagnosis and Prevention of Venous Thromboembolism during Pregnancy. J Pregnancy. 2011;2011:206858.

17. D’Uva M, Di Micco P, Strina I, et al. Venous thromboembolism and pregnancy. Journal of Blood Medicine. 2010;1:9-12. 